\title{
Real-World Outcomes in First-Line Treatment of Metastatic Castration-Resistant Prostate Cancer:The Prostate Cancer Registry
}

\author{
Simon Chowdhury ${ }^{1} \cdot$ Anders Bjartell $^{2} \cdot$ Nicolaas Lumen $^{3} \cdot$ Pablo Maroto $^{4} \cdot$ Thomas Paiss $^{5} \cdot$ Francisco Gomez-Veiga $^{6}$. \\ Alison Birtle ${ }^{7}$. Gero Kramer ${ }^{8} \cdot$ Ewa Kalinka $^{9} \cdot$ Dominique Spaëth $^{10}$. Susan Feyerabend ${ }^{11} \cdot$ Vsevolod Matveev $^{12}$. \\ Florence Lefresne ${ }^{13} \cdot$ Martin Lukac $^{14} \cdot$ Robert Wapenaar $^{15} \cdot$ Luis Costa $^{16}$
}

Published online: 4 June 2020

(c) The Author(s) 2020

\begin{abstract}
Background Metastatic prostate cancer has a 30\% 5-year survival rate despite recent therapeutic advances. There is a need to improve the clinical understanding and treatment of this disease, particularly in the real-world setting and among patients who are under-represented in clinical trials.

Objective We aimed to evaluate the characteristics and clinical outcomes of patients who received their first treatment for metastatic castration-resistant prostate cancer (mCRPC) in routine clinical practice, independent of treatment used, including subgroups with baseline cardiac disease, diabetes mellitus, or visceral metastases.

Patients and methods Prospective, noninterventional analysis of patient record data in the multicenter Prostate Cancer Registry (PCR) of men with mCRPC. The data were collected in 16 countries with the aim of recruiting more than 3000 patients between 2013 and 2016. The study end date was 9 July 2018. Data evaluated included baseline characteristics, treatment exposure, and efficacy outcomes [overall survival (OS) and time to progression (TTP)] of patients treated with abiraterone acetate plus prednisone or prednisolone (collectively, "abiraterone"), enzalutamide, or docetaxel. Descriptive outcomes are reported from the overall patient population and subgroups of patients with baseline cardiovascular disease, diabetes mellitus, or visceral metastases. The treatment effects for time to progression were compared for the overall patient population. Results The study enrollment period lasted 2.5 years, and each patient was followed for a maximum of 3 years. A total of 1874 patients in the PCR had not received previous mCRPC treatment at baseline, although they had received androgendeprivation therapy. Prevalent co-morbidities included cardiovascular disease in $65.4 \%$ and diabetes mellitus in $17.4 \%$ of patients. Baseline characteristics suggested that patients with more advanced disease received docetaxel treatment. In the overall patient population, the median time to progression with abiraterone, enzalutamide, and docetaxel as first-line mCRPC therapy was 9.6, 10.3, and 7.6 months, respectively, and median OS was 27.1, 27.1, and 27.9 months, respectively. Outcomes in the subgroups of patients with cardiovascular disease or diabetes mellitus were similar to those of the whole population in the analysis. As expected, patients with visceral metastases had shorter TTP and OS than patients in the overall population. Conclusions This analysis shows, for the first time, the effectiveness in parallel of first-line abiraterone, enzalutamide, and docetaxel in mCRPC, including in patients with co-morbidities such as cardiovascular disease or diabetes mellitus or in patients with visceral metastases. These real-world findings from the PCR provide meaningful information to help manage mCRPC, particularly in patients under-represented in clinical studies.

Trial Registration ClinicalTrials.gov identifier NCT02236637; registered September 2014.
\end{abstract}

Electronic supplementary material The online version of this article (https://doi.org/10.1007/s11523-020-00720-2) contains supplementary material, which is available to authorized users.

Simon Chowdhury

simon.chowdhury@gstt.nhs.uk

Extended author information available on the last page of the article 


\section{Key Points}

The Prostate Cancer Registry collected real-world data on the clinical characteristics, management, and outcomes of more than 3000 men with metastatic castrationresistant prostate cancer (mCRPC) from 2013-2016.

This study found that the efficacy of three major firstline treatments for mCRPC (abiraterone, enzalutamide, and docetaxel) was similar between subpopulations of patients with co-morbidities and the wider patient population.

These real-world data provide information on long-term outcomes for patients with metastatic castration-resistant prostate cancer.

\section{Background}

Prostate cancer is the second most common cancer and fifth leading cause of death from cancer in men [1]. Worldwide, an estimated 1.1 million men were diagnosed with prostate cancer in 2012, representing $15 \%$ of all cancers diagnosed in men, and the disease caused 307,000 deaths [1]. In Europe, 450,000 new cases of prostate cancer were diagnosed in 2018, and 107,000 deaths were reported [2]. Prostate cancer survival rates vary widely according to stage. Outcomes for localized prostate cancer are very good, owing to effective radical surgery or radiation therapy [3]. However, the mortality rate among men in whom the disease has progressed to metastatic prostate cancer is substantial. Although several options for treatment of metastatic castration-resistant prostate cancer (mCRPC) (abiraterone, enzalutamide, cabazitaxel, radium-223, and sipuleucel-T) have shown a survival benefit in phase 3 trials, evidence for optimal sequences or combinations is lacking [4-6]. Expert opinion is helpful for treatment decisions in routine clinical practice [7]; however, there is a substantial need to improve the understanding and management of this disease - in particular, to avoid progression to disease states in which mortality rates are high and to assess patients outside of a clinical trial setting.

The Prostate Cancer Registry (PCR) (NCT02236637) was initiated in 2013 to collect real-world data on mCRPC treatments. This is the first and largest prospective, international, observational study of patients with mCRPC. The PCR aims to document the characteristics, management, and outcomes of more than 3000 men in routine clinical practice, independent of treatment used. Enrollment took place between June 2013 and March 2016, when unprecedented progress was being made in the treatment of mCRPC in Europe. The PCR provided the opportunity to capture a wealth of data on all mCRPC treatments from this critical period in a wide range of countries and patients, including groups under-represented in clinical research. Thus, the PCR contains important data reflecting clinical practice in these challenging subgroups of patients with mCRPC, for whom data on which to base treatment decisions are often lacking.

This analysis of data in the PCR focuses on the characteristics and clinical outcomes of patients who received their first treatment for mCRPC in routine clinical practice including subgroups with baseline cardiac disease, diabetes mellitus, or visceral metastases. Specifically, baseline patient characteristics, treatment exposure, overall survival (OS), and time to progression were evaluated.

\section{Methods}

\subsection{Aim, Study Design, and Setting}

This prospective, noninterventional analysis of patient record data from the multicenter PCR of men with mCRPC was conducted to gain a greater understanding of real-world outcomes in a broad group of patients treated for mCRPC to optimize future treatment of patients with this disease. We focused on the characteristics and clinical outcomes of patients, including subgroups with baseline cardiac disease, diabetes mellitus, or visceral metastases, who received their first treatment for mCRPC during participation in the PCR. The data for the PCR were collected at 199 centers specializing in the treatment of prostate cancer by both oncologists and urologists in a range of settings (including hospitals, private clinics, outpatient departments, and universities) in 16 countries: Austria, Belgium, France, Germany, Israel, Italy, Luxembourg, Poland, Portugal, Russia, Slovenia, Spain, Sweden, Switzerland, Turkey, and the UK. Patient enrollment took place between 2013 and 2016. At the start of the PCR, abiraterone acetate and enzalutamide were not routinely available in the mCRPC setting in all 16 countries. The study duration was 5.5 years, with a maximum patient follow-up of 3 years, and was designed specifically to include more than one line of therapy where applicable. The study end date was 9 July 2018.

\subsection{Patients}

Men aged $\geq 18$ years with a histologically or cytologically confirmed diagnosis of prostate adenocarcinoma with $\mathrm{mCRPC}$ (disease progression despite testosterone $<50 \mathrm{ng} / \mathrm{dL}$ and/or androgen deprivation therapy and/or history of orchiectomy), enrolled at any time after diagnosis, under surveillance according to routine clinical practice, or who initiated a systemic treatment for mCRPC (as required) following baseline data collection at enrollment, were eligible. The 
PCR included patients treated with abiraterone acetate plus prednisone or prednisolone (collectively, "abiraterone"), enzalutamide, docetaxel, other chemotherapy, and radium-223. Only treatment groups with adequate numbers of patients $(>50)$ for reliable analysis were included. Patients treated with other chemotherapy or radium-223 were excluded because the numbers were too low.

\subsection{Variables and Data Measurement}

Treatment outcomes were duration of initial therapy and reasons for stopping initial mCRPC therapy. Appropriate treatment gaps for classifying subsequent therapy were 30 days for abiraterone or enzalutamide and 60 days for docetaxel, which included any prolonged, temporary treatment discontinuation. Efficacy outcomes were OS at 3 years after enrollment and time to progression. Criteria for disease progression on treatment (date of recording progression/assessment) were met if: (1) there was evidence of radiographic progression by investigator's assessment (Prostate Cancer Working Group 2 and Response Evaluation Criteria In Solid Tumors guidelines were recommended by the protocol); (2) there was evidence of clinical progression by investigator's assessment (date of the patient visit); (3) first-line mCRPC treatment was stopped because of progression (date treatment stopped); or (4) new mCRPC treatment was started because of progression (date new treatment began). The treatment effects for time to progression with the therapies of interest were compared for the entire analysis population. We compared treatment outcomes in the whole analysis population with those in subgroups of patients with baseline cardiovascular disease requiring treatment (including at least one of the following co-morbidities: hypertension, angina pectoris, history of myocardial infarction, arrhythmia, thromboembolic disease, transient ischemic attack, cerebrovascular accident, and/or other cardiovascular event), diabetes mellitus, or visceral metastases (defined as lesions in the liver and/or lung). These patient subgroups are typically under-represented in clinical studies. Adverse events were collected only for those patients receiving abiraterone, as a regulatory requirement.

We used observational methodology to capture data. The treating physician made treatment decisions at his or her discretion, per routine clinical practice, and only data available from clinical practice were collected. We collected prior disease history and management data at study inclusion and clinical data at study inclusion and prospectively every 3 months during routine follow-up (at least every 3 months per protocol) over the 3-year study period, with clinicaltrial levels of monitoring quality (see Supplementary Fig. $\mathrm{S} 1$ ). Because docetaxel is administered through a maximum number of cycles (typically 6-10 cycles as appropriate), outcomes influenced by duration of docetaxel treatment should not be compared with those of abiraterone or enzalutamide. Cause of death data were not collected.

\subsection{Bias}

To ensure a patient population representative of that seen in clinical practice and to reduce selection bias, the protocol stated that all consecutive patients meeting the eligibility criteria at a participating site and who agreed to participate according to local regulations were enrolled in the PCR, irrespective of their treatment.

\subsection{Study Size}

Of more than 3000 men included in the PCR, data from patients who were being treated with abiraterone, enzalutamide, or docetaxel as first-line mCRPC therapy and who had not received previous systemic $\mathrm{MCRPC}$ treatment were included in this analysis. The PCR was designed to generate data for informative purposes. Thus, this sample size was determined by pragmatic considerations, to provide data from a large population that is representative of patients with $\mathrm{mCRPC}$ in the Europe/Middle East region.

\subsection{Statistical Methods}

We only used descriptive statistics to present observed data from routine clinical practice. To manage confounding, we used propensity score methods for the comparative effectiveness analyses. We did not apply missing data imputation methods; thus, there were no sensitivity analyses, and subgroups were constructed based on medical criteria (i.e., treatment received, co-morbidities).

\section{Results}

\subsection{Patients}

At the end of study, 3003 patients were evaluated, 1874 $(62.4 \%)$ of whom had received no previous mCRPC treatment; of these, 1654 (88.3\%) started a treatment documented in the PCR (abiraterone, enzalutamide, docetaxel, other chemotherapy, radium-223, trial medication). Abiraterone, enzalutamide, or docetaxel as first-line mCRPC therapy was received by 754, 227, and 602 patients, respectively, with a median follow-up of 18.6 months. The number of patients and study flow are presented in the Supplementary Material (Fig S2). 


\subsection{Overall and First-Line mCRPC Treatment Population}

\subsubsection{Baseline Characteristics}

Patient demographics and disease and biological characteristics at study inclusion are shown in Table 1. Patients treated with docetaxel were younger than those treated with abiraterone or enzalutamide (median age [range]: 69 [44-88] vs. 76 [43-98] and 76 [53-91] years, respectively) and had a shorter median (range) time from initial diagnosis to study inclusion (2.6 [0-22] years vs. 5.0 [0-29] years and 4.5 [0-24] years, respectively). At diagnosis, de novo metastases were reported in $35.0 \%$ of patients in the abiraterone group compared with $42.3 \%$ in the enzalutamide group and $50.1 \%$ in the docetaxel group. Median (range) time from initial to metastatic diagnosis was 2.5 (0-29), 0.9 (0-21), and $0.1(0-21)$ months for the abiraterone, enzalutamide, and docetaxel groups, respectively. In addition, a Gleason score of 8-10 was reported in $51.0 \%, 60.6 \%$, and $58.4 \%$ of patients in the abiraterone, enzalutamide, and docetaxel groups, respectively.

Details regarding treatment history at study inclusion are presented in Table 2. Overall, $10.1 \%$ and $21.3 \%$ of patients had undergone either radical prostatectomy or prostate radiotherapy, respectively, since initial diagnosis (abiraterone, $11.8 \%$ and $23.1 \%$; enzalutamide, $9.5 \%$ and $23.3 \%$; docetaxel, $9.5 \%$ and $23.3 \%$; respectively) and $94.7 \%$ had received at least one prior systemic anticancer therapy since diagnosis $(95.1 \%, 93.8 \%$, and $94.2 \%$ for patients treated with abiraterone, enzalutamide, and docetaxel, respectively). Orchiectomy had been performed in $4.6 \%, 7.3 \%$, and $5.3 \%$ of patients, respectively.

\subsubsection{Treatment Exposure}

The median (95\% confidence interval [CI]) duration of treatment according to the Kaplan-Meier analysis was 11.2 (9.8-12.2) months with abiraterone, 13.0 (10.4-15.9) months with enzalutamide, and 4.8 (4.4-5.0) months with docetaxel (Supplementary Table S1). In the abiraterone, enzalutamide, and docetaxel groups, the most common reason for stopping treatment was disease progression $(72.8 \%$, $67.3 \%$, and $27.0 \%$ of patients) (Supplementary Table S2). The proportion of patients who discontinued treatment because of toxicity was $7.1 \%, 13.5 \%$, and $14.2 \%$ in these groups, and death during treatment was reported in $7.2 \%$, $11.1 \%$, and $3.8 \%$ of patients, respectively (Supplementary Table S2). In this first-line mCRPC treatment population, $48.7 \%, 43.6 \%$, and $70.8 \%$, of abiraterone-, enzalutamide-, and docetaxel-treated patients, respectively, went on to receive a subsequent treatment; the most common switch was from abiraterone to docetaxel followed by enzalutamide to docetaxel (Table 3).

\subsubsection{Efficacy Outcomes}

Kaplan-Meier estimates showed that the median (95\% confidence interval (CI)) time to progression was 9.6 (8.4-10.8), 10.3 (8.9-13.0), and 7.6 (7.0-8.0) months with abiraterone, enzalutamide, and docetaxel, respectively (Fig. 1a, Table 4). The treatment effects for time to progression comparing abiraterone versus docetaxel and enzalutamide versus docetaxel were statistically significant (all $p<0.0001$; hazard ratio $<1.0$ ), while those comparing abiraterone versus enzalutamide were not significant (all $p>0.5$; hazard ratios $>1.0$ ) (Table 5). The median (95\% CI) OS was 27.1 (25.3-28.9), 27.1 (21.8-32.7), and 27.9 (25.6-31.5) months in the abiraterone, enzalutamide, and docetaxel groups, respectively (Fig. 2a, Table 6). None of the differences between treatment groups for OS were significant (Table 7).

\subsection{Patients with Cardiovascular Disease}

\subsubsection{Baseline Characteristics}

At baseline, cardiovascular disease was reported in 504 (66.8\%), $161(70.9 \%)$, and $351(58.3 \%)$ patients in the abiraterone, enzalutamide, and docetaxel, groups, respectively; the most common cardiovascular co-morbidity was hypertension in all three treatment groups $(54.5 \%, 56.8 \%$, and $48.0 \%$, respectively), followed by specific cardiovascular co-morbidities of arrhythmia and history of myocardial infarction (Table 1). This cardiovascular disease group also had important concomitant co-morbidities for patients receiving abiraterone, enzalutamide, and docetaxel: diabetes mellitus (types 1 and 2) was reported in 101 (20.0\%), 36 (22.4\%), and $84(23.9 \%)$ patients; respiratory co-morbidities were reported in $46(9.1 \%), 23(14.3 \%)$, and $38(10.8 \%)$ patients; and neurologic co-morbidities were reported in 52 (10.3\%), 17 (10.6\%), and $37(10.5 \%)$ patients, respectively (Supplementary Table S3A).

Patients treated with abiraterone had a median (range) time from initial prostate cancer diagnosis to study inclusion of $5.3(0-29)$ years versus $4.9(0-21)$ and $3.1(0-22)$ years for the enzalutamide and docetaxel groups, respectively. Median (range) age at baseline was 77 (50-94), 77 (59-91), and 71 (47-88) years, respectively (Supplementary Table S3A), which was older than the ages reported in recent registration studies $[8,9]$. At diagnosis, 49.8\%, 59.0\%, and $56.6 \%$ of patients in the abiraterone, enzalutamide, and docetaxel groups, respectively, had a Gleason score of 8-10 (Supplementary Table S3A). Details regarding treatment history at study inclusion are presented in Supplementary Table S3B. 
Table 1 Patient demographics and disease and biological characteristics at study inclusion in the first-line mCRPC treatment population

\begin{tabular}{|c|c|c|c|c|}
\hline \multirow[t]{2}{*}{ Characteristic } & \multirow{2}{*}{$\begin{array}{l}\text { Patients who had not received } \\
\text { previous mCRPC treatment at } \\
\text { baseline } N=1874\end{array}$} & \multicolumn{3}{|c|}{ First-line treatment with } \\
\hline & & Abiraterone $N=754$ & Enzalutamide $N=227$ & Docetaxel $N=602$ \\
\hline \multicolumn{5}{|l|}{ Age, } \\
\hline Mean (SD), years & $73.1(8.58)$ & $75.3(8.20)$ & $74.9(7.87)$ & $68.8(7.70)$ \\
\hline Median (range), years & $74.0(43-98)$ & $76.0(43-98)$ & $76.0(53-91)$ & $69.0(44-88)$ \\
\hline \multicolumn{5}{|l|}{ Age group, $n(\%)$} \\
\hline$<65$ years & $314(16.8)$ & $81(10.7)$ & $24(10.6)$ & $173(28.7)$ \\
\hline $65-74$ years & $683(36.4)$ & $228(30.2)$ & $75(33.0)$ & $280(46.5)$ \\
\hline$\geq 75$ years & $877(46.8)$ & $445(59.0)$ & $128(56.4)$ & $149(24.8)$ \\
\hline \multicolumn{5}{|c|}{ Geographic distribution, $n(\%)$} \\
\hline$N$ & $1874(100.0)$ & $754(100.0)$ & $227(100.0)$ & $602(100.0)$ \\
\hline Austria & $12(0.6)$ & $2(0.2)$ & $2(0.9)$ & $2(0.3)$ \\
\hline Belgium & $129(6.9)$ & $60(8.0)$ & $25(11.0)$ & $28(4.7)$ \\
\hline France & $209(11.2)$ & $116(15.4)$ & $27(11.9)$ & $53(8.8)$ \\
\hline Germany & $240(12.8)$ & $128(17.0)$ & $19(8.4)$ & $61(10.1)$ \\
\hline Israel & $18(1.0)$ & $11(1.5)$ & 0 & $3(0.5)$ \\
\hline Italy & $238(12.7)$ & $157(20.8)$ & $2(0.9)$ & $70(11.6)$ \\
\hline Luxembourg & $8(0.4)$ & $1(0.1)$ & 0 & $6(1.0)$ \\
\hline Poland & 163 (78.7) & $1(0.1)$ & $2(0.9)$ & $122(20.3)$ \\
\hline Portugal & $78(4.2)$ & $21(2.8)$ & $2(0.9)$ & $43(7.1)$ \\
\hline Russia & $79(4.2)$ & $16(2.1)$ & $2(0.9)$ & $27(4.5)$ \\
\hline Slovenia & $22(1.2)$ & $14(1.9)$ & $2(0.9)$ & $1(0.2)$ \\
\hline Spain & $141(7.5)$ & $85(11.3)$ & $17(7.5)$ & $24(4.0)$ \\
\hline Sweden & $149(8.0)$ & $18(2.4)$ & $47(20.7)$ & $38(6.3)$ \\
\hline Switzerland & $14(0.8)$ & $6(0.8)$ & $1(0.4)$ & $7(1.2)$ \\
\hline Turkey & $61(3.3)$ & $10(1.3)$ & 0 & $47(7.8)$ \\
\hline United Kingdom & $313(16.7)$ & $108(14.3)$ & $79(34.8)$ & $70(11.6)$ \\
\hline \multicolumn{5}{|c|}{ ECOG performance status, $n(\%)$} \\
\hline$N$ & 1732 & 715 & 187 & 555 \\
\hline 0 & $746(43.1)$ & $340(47.6)$ & $99(52.9)$ & $211(38.0)$ \\
\hline 1 & $797(46.0)$ & $318(44.5)$ & $69(36.9)$ & $293(52.8)$ \\
\hline$\geq 2$ & $189(10.9)$ & $57(8.0)$ & $19(10.2)$ & $51(9.2)$ \\
\hline \multicolumn{5}{|c|}{$\begin{array}{l}\text { Gleason score at initial diagnosis, } n \\
(\%)\end{array}$} \\
\hline$N$ & 1713 & 674 & 208 & 574 \\
\hline$\leq 6$ & $216(12.6)$ & $100(14.8)$ & $25(12.0)$ & $61(10.6)$ \\
\hline 7 & $548(32.0)$ & $230(34.1)$ & $57(27.4)$ & $178(31.0)$ \\
\hline $8-10$ & $949(55.4)$ & $344(51.0)$ & $126(60.6)$ & $335(58.4)$ \\
\hline \multicolumn{5}{|c|}{ M stage at initial diagnosis, $n(\%)$} \\
\hline$N$ & 1830 & 732 & 222 & 591 \\
\hline $\mathrm{Mx}$ & $353(19.3)$ & $161(22.0)$ & $40(18.0)$ & $100(16.9)$ \\
\hline M0 & $682(37.3)$ & $315(43.0)$ & $88(39.6)$ & $195(33.0)$ \\
\hline M1, M1a, M1b, M1c & $795(43.4)$ & $256(35.0)$ & $94(42.3)$ & $296(50.1)$ \\
\hline \multicolumn{5}{|c|}{$\begin{array}{l}\text { Time from initial prostate cancer } \\
\text { diagnosis to start of the study }\end{array}$} \\
\hline$N$ & 1874 & 754 & 227 & 602 \\
\hline Median years (range) & $3.8(0-29)$ & $5.0(0-29)$ & $4.5(0-24)$ & $2.6(0-22)$ \\
\hline
\end{tabular}


Table 1 (continued)

\begin{tabular}{|c|c|c|c|c|}
\hline \multirow[t]{2}{*}{ Characteristic } & \multirow{2}{*}{$\begin{array}{l}\text { Patients who had not received } \\
\text { previous mCRPC treatment at } \\
\text { baseline } N=1874\end{array}$} & \multicolumn{3}{|c|}{ First-line treatment with } \\
\hline & & Abiraterone $N=754$ & Enzalutamide $N=227$ & Docetaxel $N=602$ \\
\hline \multicolumn{5}{|l|}{$\begin{array}{l}\text { Presence of bone metastases } \\
\quad \text { (baseline), } n(\%)\end{array}$} \\
\hline$N$ & 1396 & 550 & 184 & 451 \\
\hline Any & $1253(89.8)$ & $548(99.6)$ & $164(89.1)$ & $405(89.8)$ \\
\hline$\geq 5$ & $548(39.3)$ & $203(36.9)$ & $60(32.6)$ & $192(42.6)$ \\
\hline \multicolumn{5}{|c|}{ Visceral metastases (baseline), $n(\%)$} \\
\hline$N$ & 1484 & 612 & 163 & 517 \\
\hline Liver only & $69(4.6)$ & $13(2.1)$ & $9(5.5)$ & $39(7.5)$ \\
\hline Lung only & $96(6.5)$ & $39(6.4)$ & $7(4.3)$ & $40(7.7)$ \\
\hline Liver and lung & $17(1.1)$ & $7(1.1)$ & 0 & $9(1.7)$ \\
\hline \multicolumn{5}{|l|}{ Biological parameters } \\
\hline \multicolumn{5}{|l|}{ PSA, ng/mL } \\
\hline$N$ & 1833 & 733 & 226 & 588 \\
\hline Mean (SD) & $143.66(462.496)$ & $125.54(488.412)$ & $79.82(173.516)$ & $181.05(510.264)$ \\
\hline \multicolumn{5}{|l|}{ Lactic acid dehydrogenase, U/L } \\
\hline$N$ & 732 & 331 & 77 & 242 \\
\hline Mean (SD) & 344.5 (296.75) & $330.3(281.58)$ & $283.5(204.28)$ & $367.1(320.45)$ \\
\hline \multicolumn{5}{|l|}{ Hemoglobin, g/dL } \\
\hline$N$ & 1618 & 651 & 199 & 530 \\
\hline Mean (SD) & $12.6(1.67)$ & $12.7(1.63)$ & $12.9(1.43)$ & $12.5(1.73)$ \\
\hline \multicolumn{5}{|l|}{ Alkaline phosphatase, U/L } \\
\hline$N$ & 1365 & 575 & 191 & 414 \\
\hline Mean (SD) & $209.6(341.29)$ & $192.1(282.84)$ & $181.8(473.49)$ & $223.7(297.86)$ \\
\hline \multicolumn{5}{|c|}{ Co-morbidities requiring treatment, $n(\%)$} \\
\hline Any & $1367(72.9)$ & $566(75.1)$ & $176(77.5)$ & $398(66.1)$ \\
\hline Cardiovascular & $1221(65.2)$ & $504(66.8)$ & $161(70.9)$ & $351(58.3)$ \\
\hline Hypertension & $1002(53.5)$ & $411(54.5)$ & $129(56.8)$ & $289(48.0)$ \\
\hline Angina pectoris & $115(6.1)$ & $34(4.5)$ & $12(5.3)$ & $35(5.8)$ \\
\hline Myocardial infarction & $133(7.1)$ & $48(6.4)$ & $21(9.3)$ & $32(5.3)$ \\
\hline Arrhythmia & $154(8.2)$ & $62(8.2)$ & $22(9.7)$ & $41(6.8)$ \\
\hline Thromboembolic disease & $55(2.9)$ & $21(2.8)$ & $8(3.5)$ & $18(3.0)$ \\
\hline Cerebrovascular accident & $48(2.6)$ & $19(2.5)$ & $3(1.3)$ & $17(2.8)$ \\
\hline Transient ischemic attack & $34(1.8)$ & $14(1.9)$ & $5(2.2)$ & $9(1.5)$ \\
\hline Other cardiovascular & $300(16.0)$ & $139(18.4)$ & $39(17.2)$ & $80(13.3)$ \\
\hline Respiratory & $160(8.5)$ & $60(8.0)$ & 27 (11.9) & $48(8.0)$ \\
\hline $\begin{array}{l}\text { Chronic obstructive pulmonary } \\
\text { disease }\end{array}$ & $105(5.6)$ & $41(5.4)$ & $16(7.0)$ & $29(4.8)$ \\
\hline Other respiratory & $60(3.2)$ & $20(2.7)$ & $12(5.3)$ & $19(3.2)$ \\
\hline Renal & $143(7.6)$ & $52(6.9)$ & $24(10.6)$ & $42(7.0)$ \\
\hline Chronic renal disease & $82(4.4)$ & $31(4.1)$ & $16(7.0)$ & $21(3.5)$ \\
\hline Other renal & $62(3.3)$ & $21(2.8)$ & $8(3.5)$ & $21(3.5)$ \\
\hline Hepatic & $31(1.7)$ & $17(2.3)$ & $3(1.3)$ & $8(1.3)$ \\
\hline Chronic hepatic disease & $17(0.9)$ & $9(1.2)$ & $3(1.3)$ & $4(0.7)$ \\
\hline Other hepatic & $14(0.7)$ & $8(1.1)$ & 0 & $4(0.7)$ \\
\hline Neurologic & $157(8.4)$ & $66(8.8)$ & $17(7.5)$ & $41(6.8)$ \\
\hline Peripheral sensory impairment & $8(0.4)$ & $2(0.3)$ & 0 & $5(0.8)$ \\
\hline Memory impairment & $14(0.7)$ & $5(0.7)$ & $4(1.8)$ & $2(0.3)$ \\
\hline Cognitive disorder & $12(0.6)$ & $4(0.5)$ & $2(0.9)$ & $1(0.2)$ \\
\hline
\end{tabular}


Table 1 (continued)

\begin{tabular}{|c|c|c|c|c|}
\hline \multirow[t]{2}{*}{ Characteristic } & \multirow{2}{*}{$\begin{array}{l}\text { Patients who had not received } \\
\text { previous mCRPC treatment at } \\
\text { baseline } N=1874\end{array}$} & \multicolumn{3}{|c|}{ First-line treatment with } \\
\hline & & Abiraterone $N=754$ & Enzalutamide $N=227$ & Docetaxel $N=602$ \\
\hline Convulsion & $5(0.3)$ & $3(0.4)$ & 0 & 0 \\
\hline Other neurologic & $54(2.9)$ & $27(3.6)$ & $4(1.8)$ & $10(1.7)$ \\
\hline Infections & $11(0.6)$ & $6(0.8)$ & $3(1.3)$ & $1(0.2)$ \\
\hline Other infection & $11(0.6)$ & $6(0.8)$ & $3(1.3)$ & $1(0.2)$ \\
\hline Diabetes mellitus & $307(16.4)$ & $121(16.0)$ & 47 (20.7) & $100(16.6)$ \\
\hline Type 2 & $235(12.5)$ & 87 (11.5) & $36(15.9)$ & $82(13.6)$ \\
\hline Type 1 & $72(3.8)$ & $34(4.5)$ & $11(4.8)$ & $18(3.0)$ \\
\hline \multicolumn{5}{|l|}{ Investigations } \\
\hline Hypercholesterolemia & 276 (14.7) & $122(16.2)$ & 48 (21.1) & $62(10.3)$ \\
\hline
\end{tabular}

ECOG Eastern Cooperative Oncology Group, $M$ metastatic status, $m C R P C$ metastatic castration-resistant prostate cancer, $N$ number of evaluable patients for each specific parameter, $P S A$ prostate-specific antigen, $S D$ standard deviation

Table 2 Treatment history at study inclusion in the first-line mCRPC treatment population

\begin{tabular}{|c|c|c|c|c|}
\hline \multirow[t]{2}{*}{ Prior treatment, $n(\%)$} & \multirow{2}{*}{$\begin{array}{l}\text { Patients who had not received previous } \\
\text { mCRPC treatment at baseline } n=1874\end{array}$} & \multicolumn{3}{|c|}{ First-line treatment with } \\
\hline & & Abiraterone $n=754$ & Enzalutamide $n=227$ & Docetaxel $n=602$ \\
\hline Orchiectomy since initial diagnosis & $123(6.6)$ & $35(4.6)$ & $12(5.3)$ & $44(7.3)$ \\
\hline \multicolumn{5}{|l|}{$\begin{array}{l}\text { Radical prostatectomy/prostate-specific } \\
\text { radiotherapy since initial diagnosis }\end{array}$} \\
\hline $\begin{array}{l}\text { Both radical prostatectomy and } \\
\text { prostate-specific radiotherapy }\end{array}$ & $160(8.5)$ & $79(10.5)$ & $18(7.9)$ & $52(8.6)$ \\
\hline Radical prostatectomy only & $190(10.1)$ & $89(11.8)$ & $21(9.3)$ & $57(9.5)$ \\
\hline Prostate-specific radiotherapy only & $399(21.3)$ & $174(23.1)$ & $46(20.3)$ & $140(23.3)$ \\
\hline None & $1125(60.0)$ & $412(54.6)$ & $142(62.6)$ & $353(58.6)$ \\
\hline \multicolumn{5}{|l|}{ Prior systemic anticancer therapy } \\
\hline Any & $1774(94.7)$ & $718(95.2)$ & $213(93.8)$ & $567(94.2)$ \\
\hline Endocrine therapy & 1755 (93.6) & $710(94.2)$ & $211(93.0)$ & $562(93.4)$ \\
\hline Antiandrogen & $1569(83.7)$ & $646(85.7)$ & $188(82.8)$ & $506(84.1)$ \\
\hline GnRH agonist & $1280(68.3)$ & $474(62.9)$ & $211(93.0)$ & $434(72.1)$ \\
\hline Steroids & $194(10.4)$ & $76(10.1)$ & $22(9.7)$ & $75(12.5)$ \\
\hline GnRH antagonist & $127(6.8)$ & $64(8.5)$ & $9(4.0)$ & $39(6.5)$ \\
\hline Estrogens and derivatives & $53(2.8)$ & $28(3.7)$ & $7(3.1)$ & $5(0.8)$ \\
\hline Adrenal synthesis inhibitors & $14(0.7)$ & $6(0.8)$ & $0(0)$ & $7(1.2)$ \\
\hline Other & $6(0.3)$ & $0(0)$ & $3(1.3)$ & $2(0.3)$ \\
\hline \multicolumn{5}{|l|}{ Bone-targeted } \\
\hline Any & $455(24.3)$ & 163 (21.6) & $32(14.1)$ & $176(29.2)$ \\
\hline Zoledronic acid & $330(17.6)$ & $120(15.9)$ & $20(8.8)$ & $126(20.9)$ \\
\hline Denosumab & $103(5.5)$ & $45(6.0)$ & $10(4.4)$ & $32(5.3)$ \\
\hline Other & $55(2.9)$ & $9(1.2)$ & $6(2.6)$ & $32(5.3)$ \\
\hline Other & $28(1.5)$ & $12(1.6)$ & $5(2.2)$ & $7(1.2)$ \\
\hline
\end{tabular}

GnRH gonadotropin-releasing hormone, $m C R P C$ metastatic castration-resistant prostate cancer

\subsubsection{Treatment Exposure}

Median (95\% CI) treatment durations were 11.1 (9.5-12.7) months with abiraterone, 12.7 (9.1-15.3) months with enzalutamide, and 4.6 (4.3-5.0) months with docetaxel for typically 6-10 cycles (Supplementary Table S1); these are similar to durations observed in the equivalent overall patient population treatment groups, in which docetaxel exposure was shorter than abiraterone and enzalutamide exposures. In the abiraterone, enzalutamide, and docetaxel 
Table 3 Subsequent therapy for the first-line treatment population

\begin{tabular}{llll}
\hline$n(\%)$ & Abiraterone & Enzalutamide & Docetaxel \\
\hline $\begin{array}{l}\text { First treatment set } \\
\begin{array}{l}\text { Patients with a next } \\
\text { therapy }\end{array}\end{array}$ & 754 & 227 & 602 \\
$\begin{array}{l}\text { Therapy } \\
\text { Abiraterone }\end{array}$ & $367(48.7)$ & $99(43.6)$ & $426(70.8)$ \\
Docetaxel & $178(48.5)$ & $42(42.4)$ & - \\
Enzalutamide & $99(27.0)$ & - & $116(27.2)$ \\
Cabazitaxel & $4(1.1)$ & $2(2.0)$ & $74(17.4)$ \\
Other chemotherapy & $10(2.7)$ & $1(1.0)$ & $8(1.9)$ \\
\hline
\end{tabular}

${ }^{a}$ If treatment was interrupted for less than 30 days (abiraterone or enzalutamide) or 60 days (docetaxel or cabazitaxel), it was defined as continuing. Next therapy could be started immediately following cessation of first-line treatment

groups, the most common reason for stopping treatment was disease progression $(72.0 \%, 62.7 \%$, and $24.3 \%$ of patients, respectively) (Supplementary Table S2). Death during treatment was reported in $6.9 \%, 11.9 \%$, and $5.6 \%$ of patients in these treatment groups, respectively (Supplementary Table S2).

\subsubsection{Efficacy Outcomes}

According to Kaplan-Meier estimates, the median (95\% CI) time to progression was 9.7 (8.2-11.2), 9.8 (8.0-12.9), and 7.4 (6.6-7.9), months for abiraterone, enzalutamide, and docetaxel, respectively (Fig. 1b, Table 4). The median $(95 \%$ CI) OS was 27.4 (23.0-30.3), 26.1 (18.1-33.9), and 26.1 (23.0-29.7) months in these treatment groups, respectively (Fig. 2b, Table 6).

\subsection{Patients with Diabetes Mellitus}

\subsubsection{Baseline Characteristics}

At baseline, 121 (16.0\%), 47 (20.7\%), and 100 (16.6\%) patients had diabetes mellitus in the abiraterone, enzalutamide, and docetaxel groups, respectively; in each treatment group, a lower proportion of those with diabetes mellitus had diabetes mellitus type 1 than diabetes mellitus type 2 ( $71.9 \%$ vs. $28.1 \%, 76.6 \%$ vs. $23.4 \%$, and $82.0 \%$ vs. $18.0 \%$ in the abiraterone, enzalutamide, and docetaxel groups, respectively) (Table 1).

The median (range) time from initial diagnosis to study start was $5.7(0-29), 4.0(0-18)$, and $5.4(1-20)$ years in the abiraterone, enzalutamide, and docetaxel groups, respectively. Median (range) age at baseline was 77.0 (54-93), 77.0 (62-91), and 69.0 (51-88) years, respectively (Supplementary Table S4A). A Gleason score of 8-10 at diagnosis was reported in $47.7 \%, 55.8 \%$, and $49.0 \%$ of these treatment groups, respectively (Supplementary Table S4A). The following important concomitant co-morbidity types were reported in the diabetes mellitus group for patients receiving abiraterone, enzalutamide, and docetaxel, respectively: cardiovascular in 102 (84.3\%), 36 (76.6\%), and 85 (85.0\%) patients; neurologic in $13(10.7 \%), 4(8.5 \%)$, and $12(12.0 \%)$ patients; and renal in $10(8.3 \%), 12(25.5 \%)$, and $7(7.0 \%)$ patients (Supplementary Table S4A). Details regarding treatment history at study inclusion are presented in Supplementary Table S4B.

\subsubsection{Treatment Exposure}

Median (95\% CI) treatment duration was 11.5 (9.1-16.1), 12.2 (8.2-17.5), and 4.3 (3.6-4.9) months in the abiraterone, enzalutamide, and docetaxel (typically $6-10$ cycles) treatment groups, respectively (Supplementary Table S1), which was similar to that in treatment groups for the overall population, in which docetaxel exposure was shorter than for abiraterone and enzalutamide. The most commonly reported reason for stopping treatment in abiraterone-, enzalutamide-, and docetaxel-treated patients was disease progression $(66.3 \%, 57.9 \%$, and $24.0 \%$, respectively), followed by toxicity $(12.0 \%, 13.2 \%$, and $19.0 \%$, respectively) (Supplementary Table S2). Death during treatment was reported in $9.8 \%$, $18.4 \%$, and $5.6 \%$ of patients, respectively (Supplementary Table S2).

\subsubsection{Efficacy Outcomes}

According to Kaplan-Meier estimates, the median (95\% CI) time to progression was 12.0 (9.8-16.4), 10.3 (5.5-14.7), and 7.7 (5.7-9.0) months with abiraterone, enzalutamide, and docetaxel, respectively (Fig. 1c, Table 4). The median (95\% CI) OS was 30.8 (21.7-not estimable [NE]), 27.1 (14.7-NE), and 24.3 (16.1-29.0) months in these treatment groups, respectively (Fig. 2c, Table 6).

\subsection{Patients with Visceral Metastases}

\subsubsection{Baseline Characteristics}

Visceral metastases were reported in 59 patients (7.8\%) in the abiraterone group, 16 (7.0\%) in the enzalutamide group, and $88(14.6 \%)$ in the docetaxel group. The small number of patients with visceral metastases in the enzalutamide group meant that meaningful analyses could not be performed (Table 1). Details regarding patient demographics and disease and biological characteristics at study inclusion in patients with visceral metastases in the first-line treatment population and treatment history are presented in Supplementary Tables S5A and S5B, respectively. 
a

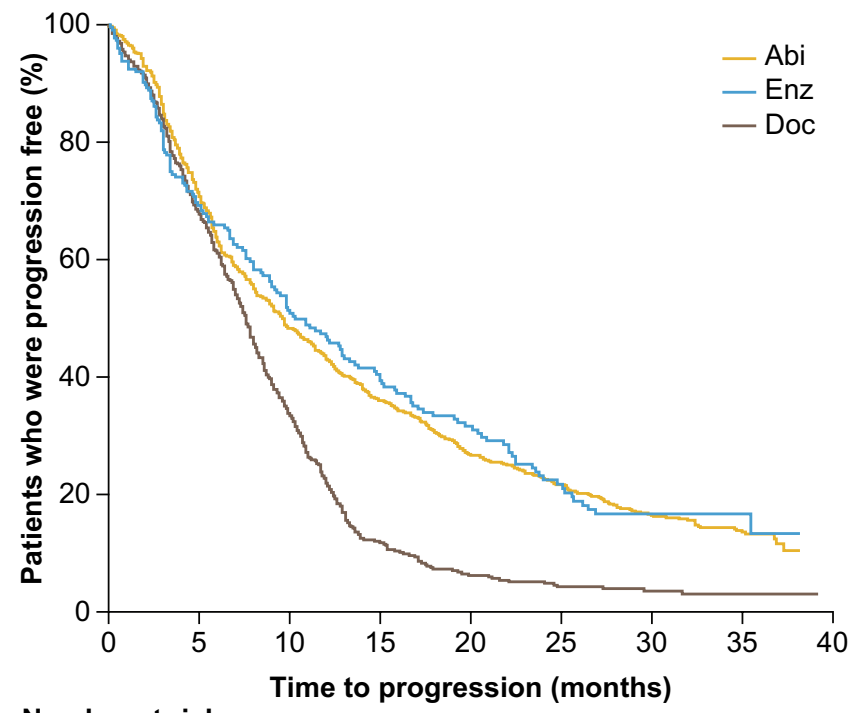

Number at risk

\begin{tabular}{|c|c|c|c|c|c|c|c|}
\hline Abi 754 & 505 & 325 & 231 & 162 & 120 & 83 & 51 \\
\hline Enz 227 & 145 & 102 & 74 & 51 & 30 & 15 & $\epsilon$ \\
\hline oc 602 & 381 & 161 & 50 & 23 & 15 & 8 & 5 \\
\hline
\end{tabular}

C

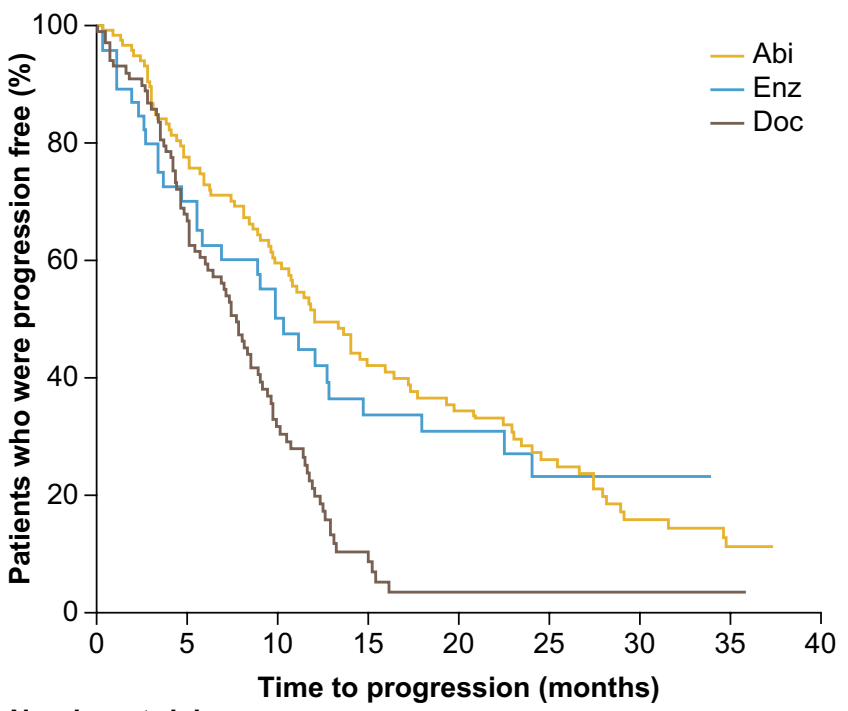

Number at risk

$\begin{array}{lccccccccc}\text { Abi } & 121 & 83 & 61 & 39 & 30 & 22 & 12 & 7 & 0 \\ \text { Enz } & 47 & 28 & 19 & 12 & 9 & 6 & 4 & 0 & 0 \\ \text { Doc } & 100 & 63 & 24 & 5 & 2 & 2 & 1 & 1 & 0\end{array}$

b

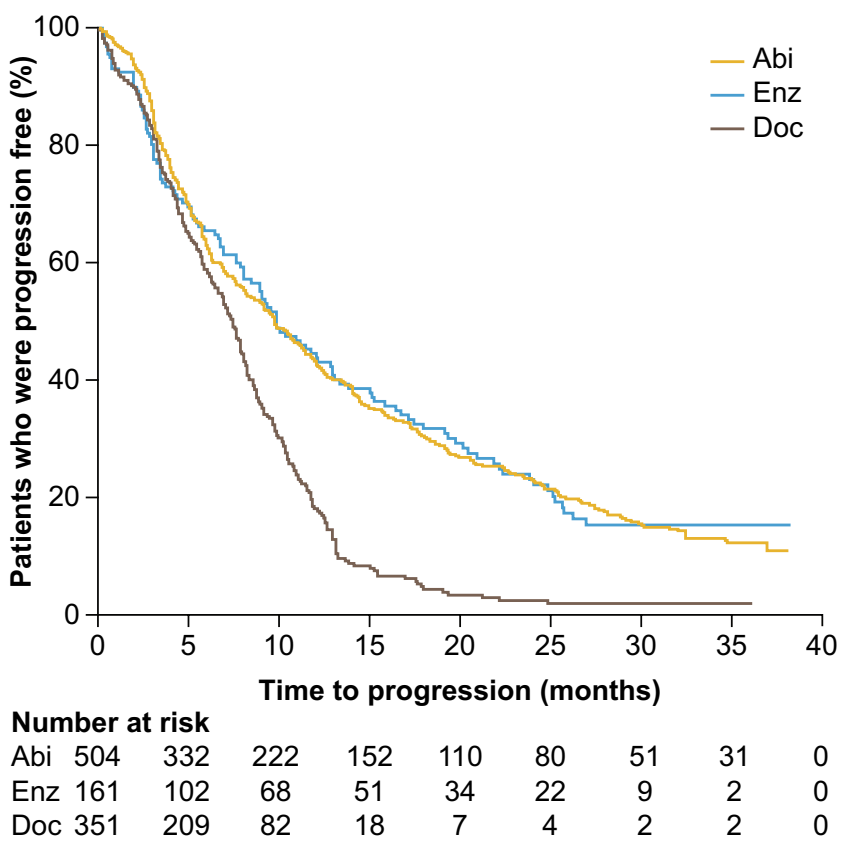

d

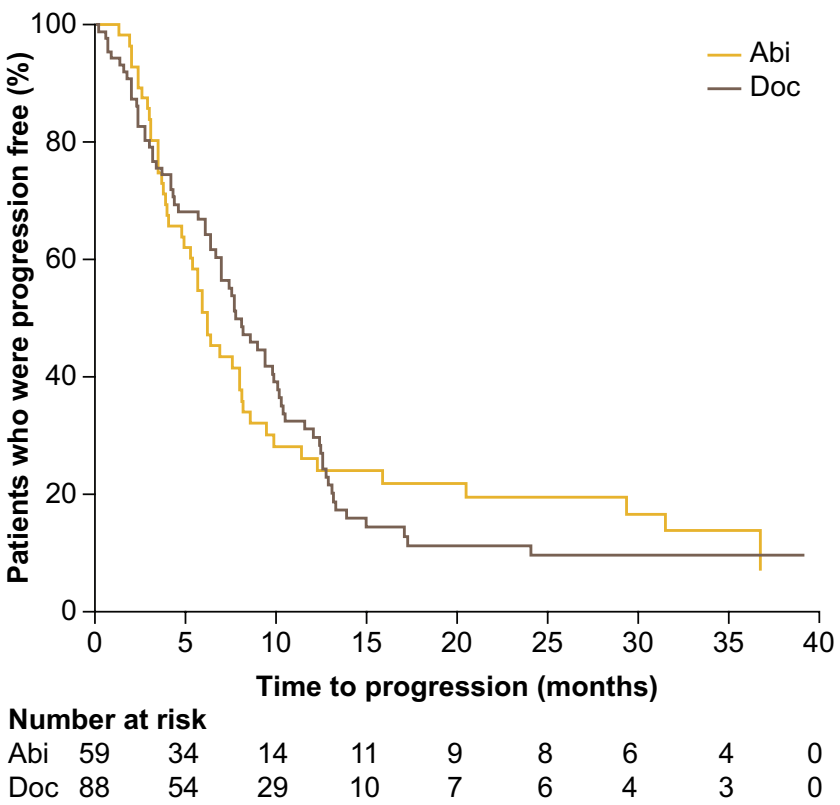

Fig. 1 Time to progression in the first-line treatment population (a), and cardiovascular (b), diabetes mellitus (c), and visceral metastases subgroups (d). Abi abiraterone, Enz enzalutamide, Doc docetaxel

\subsubsection{Treatment Exposure}

The median $(95 \% \mathrm{CI})$ treatment duration in abirateroneand docetaxel-treated patients was 7.9 (5.5-10.9) and 4.3 (3.5-5.1) months, respectively (Supplementary Table S1); the typical regimen for docetaxel treatment was $6-10$ cycles.
In this subgroup analysis, $10.4 \%$ and $11.6 \%$ of patients discontinued abiraterone and docetaxel treatment, respectively, because of toxicity; the most common reason was disease progression (70.8\% and $26.7 \%$ ), with $10.4 \%$ and $4.7 \%$ of patients dying (Supplementary Table S2). This compares to $7.4 \%, 72.8 \%$, and $7.2 \%$, for toxicity, disease progression, 
Table 4 Time to progression in the first-line treatment population, including subgroups

\begin{tabular}{|c|c|c|c|}
\hline $\begin{array}{l}\text { Kaplan-Meier estimates (patients with no progression at end } \\
\text { of Registry are censored) }\end{array}$ & Abiraterone & Enzalutamide & Docetaxel \\
\hline First-line treatment population, $N$ & 754 & 227 & 602 \\
\hline Median time to progression ${ }^{\mathrm{a}}(95 \% \mathrm{CI})$, months & $9.6(8.4-10.8)$ & $10.30(8.9-13.0)$ & $7.6(7.0-8.0)$ \\
\hline Patients with cardiovascular disease, $N$ & 504 & 161 & 351 \\
\hline Median time to progression ${ }^{\mathrm{a}}(95 \% \mathrm{CI})$, months & $9.7(8.2-11.2)$ & $9.8(8.0-12.9)$ & $7.4(6.6-7.9)$ \\
\hline Patients with diabetes mellitus, $N$ & 121 & 47 & 100 \\
\hline Median time to progression ${ }^{\mathrm{a}}(95 \% \mathrm{CI})$, months & $12.0(9.8-16.4)$ & $10.3(5.5-14.7)$ & $7.7(5.7-9.0)$ \\
\hline Patients with visceral metastases, $N$ & 59 & - & 88 \\
\hline Median time to progression ${ }^{\mathrm{a}}(95 \% \mathrm{CI})$, months & $6.2(4.8-8.1)$ & - & $7.8(6.7-9.9)$ \\
\hline
\end{tabular}

CI confidence interval

${ }^{a}$ Criteria for disease progression were met if radiographic progression (progression date is date of the assessment); if no radiographic progression documented then clinical progression per investigator's assessment (progression date is date of the patient visit) was used as the criterion. If no progression was documented using the criteria specified, then stopping medication because of progression (progression date is date that the treatment was stopped) was used as the criterion. New treatment started because of progression (progression date is date that the new treatment began) was used if previous medication was not stopped because of progression

Table 5 Unadjusted $^{\mathrm{a}}$ and adjusted ${ }^{\mathrm{b}}$ treatment effect for time to progression in the first-line treatment population

\begin{tabular}{|c|c|c|c|c|c|}
\hline Dependent variable & Unadjusted/adjusted & Comparison & $\begin{array}{l}\text { Number of } \\
\text { observations }\end{array}$ & Hazard ratio $(95 \% \mathrm{CI})$ & $p$ value \\
\hline \multicolumn{6}{|c|}{ Unadjusted and adjusted treatment effect, time to progression, abiraterone vs. docetaxel (first line) } \\
\hline Time to progression & Unadjusted & Abiraterone vs. docetaxel & $n=1356$ & $0.599(0.529-0.678)$ & $<0.0001$ \\
\hline Time to progression & Unadjusted & Abiraterone vs. docetaxel & $n=1049$ & $0.597(0.519-0.686)$ & $<0.0001$ \\
\hline Time to progression & Adjusted & Abiraterone vs. docetaxel & $n=1049$ & $0.685(0.586-0.800)$ & $<0.0001$ \\
\hline \multicolumn{6}{|c|}{ Unadjusted and adjusted treatment effect, time to progression, enzalutamide vs. docetaxel (first line) } \\
\hline Time to progression & Unadjusted & Enzalutamide vs. docetaxel & $n=829$ & $0.548(0.456-0.657)$ & $<0.0001$ \\
\hline Time to progression & Unadjusted & Enzalutamide vs. docetaxel & $n=674$ & $0.556(0.455-0.678)$ & $<0.0001$ \\
\hline Time to progression & Adjusted & Enzalutamide vs. docetaxel & $n=674$ & $0.564(0.455-0.698)$ & $<0.0001$ \\
\hline \multicolumn{6}{|c|}{ Unadjusted and adjusted treatment effect, time to progression, abiraterone vs. enzalutamide (first line) } \\
\hline Time to progression & Unadjusted & Abiraterone vs. enzalutamide & $n=981$ & $1.031(0.867-1.226)$ & 0.7302 \\
\hline Time to progression & Unadjusted & Abiraterone vs. enzalutamide & $n=751$ & $1.009(0.833-1.222)$ & 0.9277 \\
\hline Time to progression & Adjusted & Abiraterone vs. enzalutamide & $n=751$ & $1.040(0.851-1.271)$ & 0.7000 \\
\hline
\end{tabular}

CI confidence interval

${ }^{\text {a }}$ The unadjusted analysis is a Cox proportional hazards model with treatment as only predictor

${ }^{\mathrm{b}}$ The adjusted analysis is similar to the unadjusted but is based on the propensity score method; potential confounders are: age, log of time from diagnosis to castration resistance, log of time from diagnosis to metastasis, log of time from metastasis to study start, log alkaline phosphatase, $\log$ prostate-specific antigen, log hemoglobin, Gleason score, diabetes, use of strong analgesics, use of weak analgesics, cardiovascular disease, ECOG status 2-3, ECOG status unknown, prior radical prostatectomy, $\mathrm{T}$ class 3, T class 4, T class unknown, $\mathrm{N}$ class $1, \mathrm{~N}$ class unknown, $\mathrm{M}$ class 1 , M class unknown, bone lesions $5-20$, bone lesions $>20$, and bone lesions unknown

and death, respectively, during treatment in the whole abiraterone treatment group and $14.2 \%, 27.0 \%$, and $3.8 \%$ in the whole docetaxel group (Supplementary Table S2).

\subsubsection{Efficacy Outcomes}

In patients with visceral metastases, the median $(95 \% \mathrm{CI})$ time to progression was $6.2(4.8-8.1)$ and 7.8 (6.7-9.9) months with abiraterone and docetaxel, respectively (Fig. 1d, Table 4). The median (95\% CI) OS was 17.40 (13.60-22.70) and 20.40 (15.10-26.10) months in abiraterone- and docetaxel-treated patients, respectively - shorter than in the respective overall patient populations (Fig. 2d, Table 6).

\section{Discussion}

The data in the PCR provide the first opportunity to evaluate characteristics and outcomes of the largest cohort of patients with mCRPC receiving first-line treatment in 
a

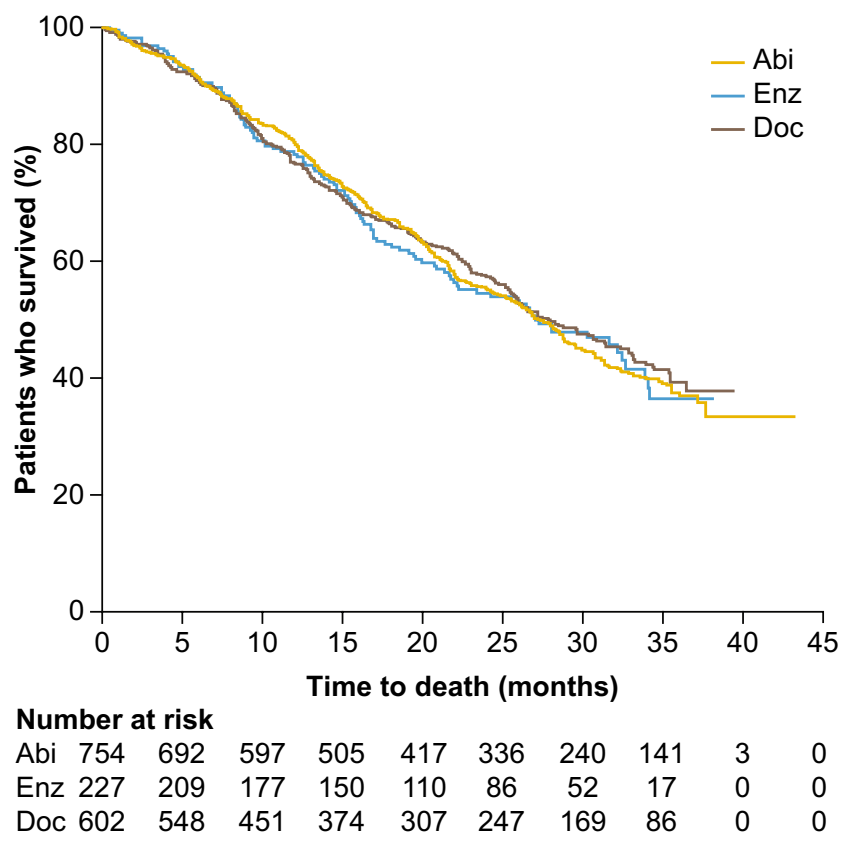

C

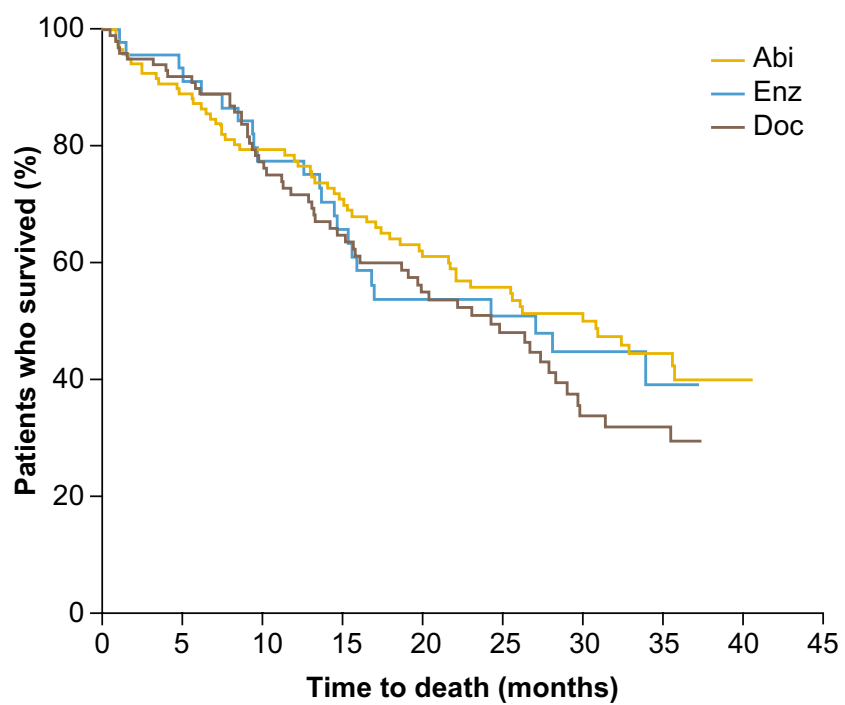

Number at risk

$\begin{array}{lllllllllll}\text { Abi } & 121 & 103 & 87 & 74 & 60 & 51 & 39 & 23 & 1 & 0\end{array}$

$\begin{array}{lllllllllll}\text { Enz } & 47 & 42 & 34 & 28 & 20 & 17 & 11 & 5 & 0 & 0\end{array}$

$\begin{array}{llllllllll}\text { Doc } 100 & 92 & 70 & 55 & 43 & 32 & 18 & 13 & 0 & 0\end{array}$ b

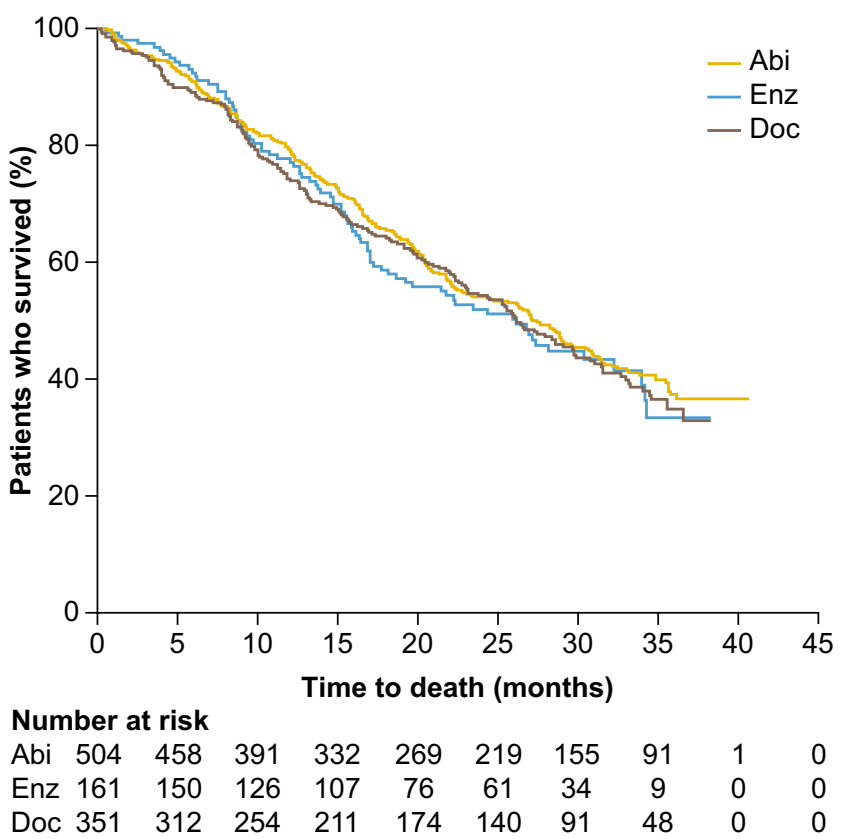

d

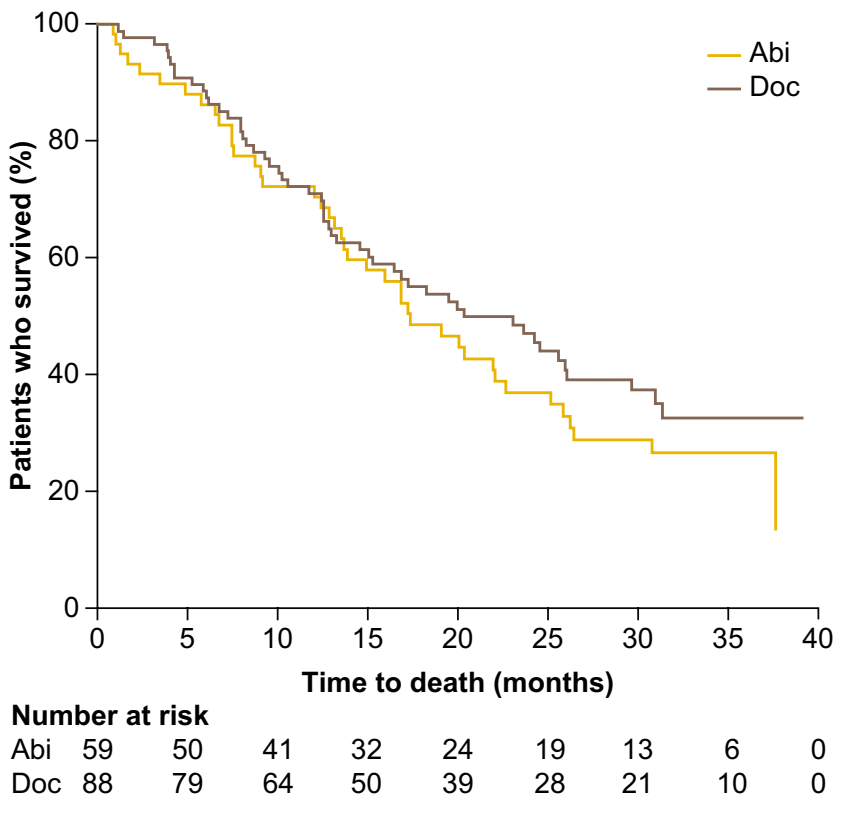

Fig. 2 Overall survival in the first-line treatment population (a), and cardiovascular (b), diabetes mellitus (c), and visceral metastases subgroups (d). Abi abiraterone, Enz enzalutamide, Doc docetaxel

the real-world setting. Notably, they encompass information from patients from 16 countries included in the PCR between 2013 and 2016.

In this analysis of a real-world setting, patients treated with docetaxel as their first-line systemic mCRPC treatment were younger than those treated with abiraterone or enzalutamide and had a shorter time from initial diagnosis to inclusion in the study. In addition, a higher proportion of patients in the docetaxel group had an Eastern Cooperative Oncology Group score of 1, visceral metastases, five or more bone metastases at baseline, and had de novo metastases at initial diagnosis. Biological parameters of prostate-specific antigen, lactate dehydrogenase, and alkaline phosphatase levels also tended to be higher in patients 
Table 6 Overall survival in the first-line treatment population, including subgroups

\begin{tabular}{llll}
\hline & Abiraterone & Enzalutamide & Docetaxel \\
\hline First-line treatment population, $N$ & 754 & 227 & 602 \\
Median overall survival (95\% CI), months & $27.1(25.3-28.9)$ & $27.1(21.8-32.7)$ & $27.9(25.6-31.5)$ \\
Patients with cardiovascular disease, $N$ & 504 & 161 & 351 \\
Median overall survival (95\% CI), months & $27.4(23.0-30.3)$ & $26.1(18.1-33.9)$ & $26.1(23.0-29.7)$ \\
Patients with diabetes mellitus, $N$ & 121 & 47 & 100 \\
Median overall survival (95\% CI), months & $30.8(21.7-\mathrm{NE})$ & $27.1(14.7-\mathrm{NE})$ & $24.3(16.1-29.0)$ \\
Patients with visceral metastases, $N$ & 59 & - & 88 \\
Median overall survival (95\% CI), months & $17.40(13.60-22.70)$ & - & $20.40(15.10-26.10)$ \\
\hline
\end{tabular}

Patients alive at end of Registry are censored

$C I$ confidence interval, $N$ number of evaluable patients for each specific parameter, $N E$ not estimable

Table 7 Unadjusted $^{\mathrm{a}}$ and adjusted $^{\mathrm{b}}$ treatment effect for overall survival in the first-line treatment population

\begin{tabular}{|c|c|c|c|c|c|}
\hline Dependent variable & Unadjusted/adjusted & Comparison & $\begin{array}{l}\text { Number of } \\
\text { observations }\end{array}$ & Hazard ratio $(95 \% \mathrm{CI})$ & $p$ value \\
\hline \multicolumn{6}{|c|}{ Unadjusted and adjusted treatment effect, overall survival, abiraterone vs. docetaxel (first line) } \\
\hline Overall survival & Unadjusted & Abiraterone vs. docetaxel & $n=1356$ & $1.035(0.891-1.202)$ & 0.6520 \\
\hline Overall survival & Unadjusted & Abiraterone vs. docetaxel & $n=1049$ & $0.994(0.841-1.176)$ & 0.9462 \\
\hline Overall survival & Adjusted & Abiraterone vs. docetaxel & $n=1049$ & $1.105(0.920-1.328)$ & 0.2843 \\
\hline \multicolumn{6}{|c|}{ Unadjusted and adjusted treatment effect, overall survival, enzalutamide vs. docetaxel (first line) } \\
\hline Overall survival & Unadjusted & Enzalutamide vs. docetaxel & $n=829$ & $1.047(0.843-1.301)$ & 0.6763 \\
\hline Overall survival & Unadjusted & Enzalutamide vs. docetaxel & $n=674$ & $1.064(0.841-1.347)$ & 0.6039 \\
\hline Overall survival & Adjusted & Enzalutamide vs. docetaxel & $n=674$ & $1.075(0.837-1.380)$ & 0.5727 \\
\hline \multicolumn{6}{|c|}{ Unadjusted and adjusted treatment effect, overall survival, abiraterone vs. enzalutamide (first line) } \\
\hline Overall survival & Unadjusted & Abiraterone vs. enzalutamide & $n=981$ & $0.983(0.798-1.211)$ & 0.8741 \\
\hline Overall survival & Unadjusted & Abiraterone vs. enzalutamide & $n=751$ & $0.927(0.737-1.165)$ & 0.5147 \\
\hline Overall survival & Adjusted & Abiraterone vs. enzalutamide & $n=751$ & $1.000(0.788-1.270)$ & 0.9986 \\
\hline
\end{tabular}

CI confidence interval

${ }^{a}$ The unadjusted analysis is a Cox proportional hazards model with treatment as only predictor

${ }^{\mathrm{b}}$ The adjusted analysis is similar to the unadjusted, but is based on the propensity score method; potential confounders are: age, log of time from diagnosis to castration resistance, $\log$ of time from diagnosis to metastasis, log of time from metastasis to study start, log alkaline phosphatase, $\log$ prostate-specific antigen, log hemoglobin, Gleason score, diabetes, use of strong analgesics, use of weak analgesics, cardiovascular disease, ECOG status 2-3, ECOG status unknown, prior radical prostatectomy, T class 3 , T class 4 , T class unknown, $\mathrm{N}$ class $1, N$ class unknown, $\mathrm{M}$ class $1, M$ class unknown, bone lesions $5-20$, bone lesions $>20$, and bone lesions unknown

treated with docetaxel than in those who received the other treatment options. Together, these findings suggest that the patients treated with docetaxel had more advanced disease and poorer prognosis. These differences in baseline characteristics are not unexpected, given the observational methodology used to capture the treatment decisions made at the discretion of the treating physician. The clinical efficacy and safety of docetaxel therapy in prostate cancer was achieved with a recommended dose of $75 \mathrm{mg} / \mathrm{m}^{2}$ every 3 weeks over 10 cycles [10]; the median duration of docetaxel treatment was approximately 4 months in the patient groups in the analysis. In contrast, the recommended dose of abiraterone was $1000 \mathrm{mg}$ once daily [11], and the usual dose of enzalutamide is $160 \mathrm{mg}$ taken at the same time once daily [12]. In general, treatment duration was not influenced by the presence of cardiovascular disease or diabetes mellitus in this study.

In this real-world analysis, which includes patients who are under-represented in prostate cancer clinical trials, median OS of those enrolled in the PCR treated with first-line abiraterone, enzalutamide, or docetaxel as mCRPC therapy was $27.1,27.1$, or 27.9 months, respectively, compared with a median OS of 34.7 months for patients treated with abiraterone acetate in the phase 3 COU-AA-302 study 
in asymptomatic or mildly symptomatic patients with chemotherapy-naive prostate cancer [13] and 18.9 months with three-times-weekly docetaxel in patients with metastatic hormone-refractory prostate cancer in the phase 3 TAX 327 study [9]. However, at the time of the TAX 327 study, subsequent treatment options for mCRPC were limited, which may have influenced the OS rates in this study. In the FIRSTANA study, which evaluated cabazitaxel versus docetaxel treatment in chemotherapy-naïve patients with mCRPC, median OS was 24.3 months for docetaxel [14], which is similar to our findings. This study was initiated at a time when novel hormonal treatments were being used clinically to treat mCRPC after docetaxel.

In the phase 3 PREVAIL study of enzalutamide in patients with disease progression despite androgendeprivation therapy, median OS was longer, at 32.4 months [8]. However, the older age, presence of certain baseline characteristics (visceral metastases, ECOG status of 2 and above), and longer disease duration before treatment of patients in the PCR compared with those in the clinical trials is likely to have affected treatment outcomes. Similarly, shorter OS in renal cancer has been reported for sunitinib in the non-clinical trial setting (18.7 months) compared with the first-line clinical setting (26.4 months) [15]. Treatment outcomes for mCRPC did not appear to be compromised by cardiovascular disease and diabetes mellitus in the present study; however, in patients with visceral metastases, OS was approximately 7 months shorter than in the overall population.

Data from the PCR provide information on patient subgroups that are poorly represented in pivotal trials. Notably, the PCR provides information on many patients with cardiovascular co-morbidities and patients with both cardiovascular and diabetic co-morbidities. The PCR does not provide information on the severity of the co-morbidities or whether patients' conditions related to co-morbidities at baseline were well controlled by concomitant medications at baseline, which may affect their outcomes. Other than disease progression, toxicity was the next most common reason for stopping systemic mCRPC treatment in patients with cardiovascular disease $(8.9 \%$ and $16.7 \%$ of patients treated with abiraterone and enzalutamide, respectively). This was also a reason for stopping treatment in patients with diabetes mellitus (12.0\% and $13.2 \%$ stopped abiraterone and enzalutamide, respectively). In addition, the higher incidence of treatment-limiting toxicity in patients with diabetes mellitus compared with the overall population and in patients with cardiovascular disease suggests that extra care should be taken in treating these men.

This study benefits from a number of strengths, including robust monitoring providing clinical trial-level quality data, the size and scope of the Registry, allowing for a large amount of data to be collected, inclusion of patient subgroups under-represented in clinical trials, and a duration that allows mature data to be collected during a period that has seen a number of advances in mCRPC treatment. Furthermore, this is the largest data set on abiraterone treatment in patients with CRPC with visceral metastases in the real-world setting. However, several limitations exist. This is an observational study, and there is no randomization to allow direct treatment comparisons. In addition, because treatment followed clinical practice, duration of exposure varied between treatments based on treatment cycles. Thus, the results for duration of treatment and reason for stopping treatment or number of deaths during the treatment period cannot be compared between docetaxel and abiraterone or docetaxel and enzalutamide because of the possible influence of the dosing regimens of the drugs; docetaxel has a shorter dosing regimen, being administered in 6-10 cycles, compared with the daily regimens of abiraterone acetate and enzalutamide, which are administered until the point of disease progression. Data were also collected at routine clinical visits, which may not have occurred at regular intervals for all patients. The commercial availability of the treatments in participating countries, different treatment practices and settings, and timing of enrollment should also be taken into consideration; the treatment options available in each country of the PCR varied according to availability of different drugs, which may have influenced findings.

\section{Conclusions}

This analysis shows for the first time the effectiveness in parallel of three major treatments in first-line mCRPC therapy: abiraterone, enzalutamide, and docetaxel. More importantly, the efficacy of these treatments was similar among subpopulations of patients with co-morbidities such as cardiovascular disease or diabetes mellitus compared with the wider patient group. For the subpopulation of patients with visceral metastases, outcomes were as expected considering the poor prognosis for this subgroup. This real-world analysis of long-term efficacy outcomes of patients in the PCR provides meaningful data to support physicians in managing different patient groups with mCRPC, which is associated with poor prognosis and mortality. Because the PCR includes patients from several countries and represents real-world practice, these results apply to most men with mCRPC.

Acknowledgements The authors acknowledge Edwin Martens who provided statistical support for the Propensity Score method analysis, Emma Lee who facilitated author liaison and project support for this manuscript, and Parexel, which provided editorial assistance in the development of this manuscript.

Author contributions SC was involved in data collection, had full access to all the data in the study, provided review of the manuscript, 
and had final responsibility for the decision to submit for publication. A Bjartell was involved in data collection, contributed to data interpretation, reviewed the manuscript, and participated as co-investigator. NL was involved in patient recruitment/data collection, data interpretation, and reviewing the manuscript. PM was involved in data collection, contributed to data analysis and interpretation and review of the manuscript. TP was involved in data collection and contributed to review of manuscript drafts. FGV was involved in data collection, contributed to data interpretation, reviewed the manuscript, and participated as coinvestigator. A Birtle was involved in data collection and contributed to review of manuscript drafts. GK was involved in data collection and contributed to review of manuscript drafts. EK was involved in data collection, data interpretation, and reviewing the manuscript. DS was involved in data collection, contributed to study design, data analysis, data interpretation, and reviewing the manuscript. SF was involved in data collection and contributed to review of manuscript drafts. VM was involved in data collection and contributed to review of manuscript drafts. FL and RW contributed to study design, data analysis, data interpretation, and reviewing the manuscript. ML contributed to data analysis, data interpretation, and reviewing the manuscript. LC was involved in data collection, contributed to data interpretation, reviewed the manuscript, and participated as a co-investigator.

Funding This study was funded by Janssen EMEA. Janssen EMEA contributed to the study design, the collection, analysis, and interpretation of data, the writing of the report, and the decision to submit the paper for publication.

\section{Compliance with ethical standards}

Conflicts of interest Simon Chowdhury has participated in speaker bureaus and acted as a consultant for Johnson \& Johnson, Astellas, Sanofi, and Clovis and has received research grants from Clovis. Anders Bjartell has received remuneration from Janssen, Astellas, and Bayer for lectures and for participation in advisory boards. Nicolaas Lumen has participated in advisory boards for Astellas, Janssen, and Bayer, receives grants from Astellas, Janssen, Bayer, Sanofi, Ferring, and Ipsen, and receives speaker honoraria from Bayer, Astellas, Janssen, GSK, and Ipsen. Pablo Maroto has participated in advisory boards for Janssen, Astellas, and Bayer. Thomas Paiss reports no conflicts of interest. Francisco Gomez-Veiga has received remuneration from Bayer, Astellas, Janssen, and Amgen for lectures and for participation in advisory boards. Alison Birtle has participated in advisory boards and provided educational meeting support for Janssen, Astellas, Sanofi Genzyme, Bayer, and Roche. Gero Kramer reports no conflicts of interest. Ewa Kalinka has received honoraria from Janssen and Medivation/Pfizer. Dominique Spaëth reports no conflicts of interests. Susan Feyerabend reports no conflicts of interest. Vsevolod Matveev participated in advisory boards for and received speaker honoraria from Astellas, Bayer, Janssen, and Sanofi. Florence Lefresne is an employee of Janssen Pharmaceutica N.V. and holds stock in Johnson \& Johnson. Martin Lukac is an employee of Parexel International Czech Republic s.r.o, on behalf of Janssen Pharmaceutica N.V., Beerse, Belgium. Robert Wapenaar is an employee of Janssen-Cilag B.V. and holds stock in Johnson \& Johnson. Luis Costa has participated in advisory boards for Janssen, Astellas, and Bayer, and has received speaker honoraria from Janssen and Bayer.

Ethics approval and consent to participate The study was approved by the ethics committees of every country that participated. The study was conducted in accordance with the Declaration of Helsinki.

Availability of data and material The data sharing policy of Janssen Pharmaceutical Companies of Johnson \& Johnson is available at https ://www.janssen.com/clinical-trials/transparency. As noted on this site, requests for access to the study data can be submitted through Yale Open Data Access (YODA) Project site at https://yoda.yale.edu.

Open Access This article is licensed under a Creative Commons Attribution-NonCommercial 4.0 International License, which permits any non-commercial use, sharing, adaptation, distribution and reproduction in any medium or format, as long as you give appropriate credit to the original author(s) and the source, provide a link to the Creative Commons licence, and indicate if changes were made. The images or other third party material in this article are included in the article's Creative Commons licence, unless indicated otherwise in a credit line to the material. If material is not included in the article's Creative Commons licence and your intended use is not permitted by statutory regulation or exceeds the permitted use, you will need to obtain permission directly from the copyright holder. To view a copy of this licence, visit http://creativecommons.org/licenses/by-nc/4.0/.

\section{References}

1. World Health Organization. Globocan 2018: all cancers. https:// gco.iarc.fr/today/data/factsheets/cancers/39-All-cancers-fact-sheet .pdf. Last update: 2018. Accessed May 17, 2019.

2. Ferlay J, Colombet M, Soerjomataram I, Dyba T, Randi G, Bettio $\mathrm{M}$, et al. Cancer incidence and mortality patterns in Europe: estimates for 40 countries and 25 major cancers in 2018. Eur J Cancer. 2018;103:356-87.

3. American Society of Clinical Oncology. Prostate cancer: statistics. https://www.cancer.net/cancer-types/prostate-cancer/statistics. Last update: Jan 2019. Accessed May 17, 2019.

4. Basch E, Loblaw DA, Oliver TK, Carducci M, Chen RC, Frame $\mathrm{JN}$, et al. Systemic therapy in men with metastatic castrationresistant prostate cancer: American Society of Clinical Oncology and Cancer Care Ontario clinical practice guideline. J Clin Oncol. 2014;32:3436-48.

5. Lorente D, Mateo J, Perez-Lopez R, de Bono JS, Attard G. Sequencing of agents in castration-resistant prostate cancer. Lancet Oncol. 2015;16:e279-e292292.

6. Nuhn P, De Bono JS, Fizazi K, Freedland SJ, Grilli M, Kantoff PW, et al. Update on systemic prostate cancer therapies: management of metastatic castration-resistant prostate cancer in the era of precision oncology. Eur Urol. 2019;75:88-99.

7. Gillessen S, Attard G, Beer TM, Beltran H, Bossi A, Bristow R, et al. Management of patients with advanced prostate cancer: the report of the Advanced Prostate Cancer Consensus Conference APCCC 2017. Eur Urol. 2018;73:178-211.

8. Beer TM, Armstrong AJ, Rathkopf DE, Loriot Y, Sternberg CN, Higano CS, et al. Enzalutamide in metastatic prostate cancer before chemotherapy. N Engl J Med. 2014;371:424-33.

9. Tannock IF, de Wit R, Berry WR, Horti J, Pluzanska A, Chi KN, et al. Docetaxel plus prednisone or mitoxantrone plus prednisone for advanced prostate cancer. N Engl J Med. 2004;351:1502-12.

10. Taxotere SPC. https://www.ema.europa.eu/documents/produ ct-information/taxotere-epar-product-information_en.pdf. Last update: Accessed May 17, 2019.

11. Zytiga SPC. https://www.medicines.org.uk/emc/files/pil.2381.pdf. Last update: Feb 2019. Accessed May 17, 2019.

12. Xtandi SPC. https://www.medicines.org.uk/emc/files/pil.3203.pdf. Last update: Oct 2018. Accessed May 17, 2019.

13. Ryan CJ, Smith MR, Fizazi K, Saad F, Mulders PF, Sternberg $\mathrm{CN}$, et al. Abiraterone acetate plus prednisone versus placebo plus prednisone in chemotherapy-naive men with metastatic castrationresistant prostate cancer (COU-AA-302): final overall survival 
analysis of a randomised, double-blind, placebo-controlled phase 3 study. Lancet Oncol. 2015;16:152-60.

14. Oudard S, Fizazi K, Sengelov L, Daugaard G, Saad F, Hansen S, et al. Cabazitaxel versus docetaxel as first-line therapy for patients with metastatic castration-resistant prostate cancer: a randomized phase III trial-FIRSTANA. J Clin Oncol. 2017;35:3189-97.
15. Gore ME, Szczylik C, Porta C, Bracarda S, Bjarnason GA, Oudard $\mathrm{S}$, et al. Final results from the large sunitinib global expandedaccess trial in metastatic renal cell carcinoma. Br J Cancer. 2015;113:12-9.

\section{Affiliations}

\section{Simon Chowdhury ${ }^{1} \cdot$ Anders Bjartell $^{2} \cdot$ Nicolaas Lumen $^{3} \cdot$ Pablo Maroto $^{4} \cdot$ Thomas Paiss $^{5} \cdot$ Francisco Gomez-Veiga $^{6}$. Alison Birtle ${ }^{7}$. Gero Kramer ${ }^{8}$. Ewa Kalinka ${ }^{9}$. Dominique Spaëth ${ }^{10}$. Susan Feyerabend ${ }^{11} \cdot$ Vsevolod Matveev $^{12}$. Florence Lefresne ${ }^{13} \cdot$ Martin Lukac $^{14} \cdot$ Robert Wapenaar $^{15} \cdot$ Luis Costa $^{16}$}

1 Guy's and St Thomas' NHS Foundation Trust and Sarah Cannon Research Institute, Westminster Bridge Rd, Lambeth, London SE1 7EH, UK

2 Department of Urology, Skåne University Hospital Malmö, Malmö, Sweden

3 Department of Urology, Ghent University Hospital, C. Heymanslaan 10, 9000 Ghent, Belgium

4 Department of Medical Oncology, Hospital de la Santa Creu i Sant Pau, Autonomous University of Barcelona, 08025 Barcelona, Spain

5 Urologie Team Ulm, Ulm, Germany

6 Urology Department and Kidney Transplant Unit, Translational Research Group of Urology GITUR-IBSAL, Salamanca University Hospital, Salamanca, Spain

7 Royal Preston Hospital, Preston, UK

8 Department of Urology, Medical University of Vienna, Vienna, Austria
9 Clinic of Oncology, Polish Mother's Memorial HospitalResearch Institute, Lodz, Poland

10 Centre d'Oncologie de Gentilly, Nancy, France

11 Studienpraxis Urologie, Nürtingen, Germany

12 N.N. Blokhin National Cancer Research Center, Moscow, Russia

13 EMEA Oncology, Janssen Pharmaceutica N.V., Beerse, Belgium

14 Parexel International Czech Republic s.r.o, on behalf of Janssen Pharmaceutica N.V., Beerse, Belgium

15 Janssen-Cilag B.V., Breda, the Netherlands

16 Oncology Division, Hospital de Santa Maria, Instituto de Medicina Molecular, Faculdade de Medicina, Universidade de Lisboa, 1649-035 Lisbon, Portugal 